\title{
Early earthquake detection capabilities of different types of future-generation gravity gradiometers
}

\author{
Tomofumi Shimoda ${ }_{3}^{1, *}$, Kévin Juhel ${ }^{2,3}$, Jean-Paul Ampuero ${ }^{4}$, Jean-Paul Montagner ${ }^{2}$, \\ \& Matteo Barsuglia ${ }^{3}$ \\ ${ }^{1}$ Department of Physics, University of Tokyo, 7-3-1, Hongo, Bunkyo-ku, Tokyo, Japan \\ 2 Université de Paris, Institut de physique du globe de Paris, CNRS, F-75005 Paris, France \\ ${ }^{3}$ Université de Paris, CNRS, Astroparticule et Cosmologie, F-75013 Paris, France \\ ${ }^{4}$ Université Côte d'Azur, IRD, CNRS, Observatoire de la Côte d'Azur, Géoazur, Sophia Antipolis, France \\ *E-mail : shimoda@granite.phys.s.u-tokyo.ac.jp
}

\begin{abstract}
SUMMARY
Since gravity propagates at the speed of light, gravity perturbations induced by earthquake deformation have the potential to enable faster alerts than the current earthquake early warning systems based on seismic waves. Additionally, for large earthquakes $\left(M_{w}>8\right)$, gravity signals may allow for a more reliable magnitude estimation than seismic-based methods. Prompt elastogravity signals induced by earthquakes of magnitude larger than 7.9 have been previously detected with seismic arrays and superconducting gravimeters. For smaller earthquakes, down to $M_{w} \simeq 7$, it has been proposed that detection should be based on measurements of the gradient of the gravitational field, in order to mitigate seismic vibration noise and to avoid the canceling effect of the ground motions induced by gravity signals. Here we simulate the five independent components of the gravity gradient signals induced by earthquakes of different focal mechanisms. We study their spatial amplitude distribution to determine what kind of detectors is preferred (which components of the gravity gradient are more informative), how detectors should be arranged, and how earthquake source parameters can be estimated. The results show that early earthquake detections, within 10 seconds of the rupture onset, using only the horizontal gravity strain components are achievable up to about $140 \mathrm{~km}$ distance from the epicenter. Depending on the earthquake focal mechanism and on the detector location, additional measurement of the vertical gravity strain components can enhance the detectable range by 10-20 $\mathrm{km}$. These results are essential for the design of gravity-based earthquake early warning systems.
\end{abstract}

Key words: earthquake early warning, prompt gravity signals, gravity gradiometer

\section{INTRODUCTION}

Earthquake early warning systems (EEWS) are essential tools for disaster risk reduction. Current systems detect earthquakes and estimate their source parameters based on the initial P-waves, which precede the most damaging shaking carried by S-waves (Allen \& Melgar, 2019). However, the promptness of current systems is limited by the fact that P-waves, the information carrier, are less than twice as fast as S-waves, the damage carrier: earthquake detection and alert take a substantial portion of the travel time of the hazard, especially if seismometers are not located near the epicenter. For offshore earthquakes, the Japanese EEWS has been improved by using cabled ocean bottom seismometers, although at a high cost and difficulty of maintenance, and elsewhere the emerging technology of Distributed Acoustic Sensing on seafloor fiber-optic cables is being explored (Sladen et al., 2019).

Recently a new method of earthquake early warning has been proposed that is based on "prompt gravity signals", the transient gravity perturbations caused by the mass redistribution induced by deformation during earthquakes (Harms et al., 2015; Juhel et al., 2018). Since gravity propagates at the speed of light, prompt gravity signals are a faster messenger for earthquake detection than $\mathrm{P}$ waves. In addition, their amplitude is directly linked to the earthquake seismic moment and, in contrast to seismic waves on broadband seismometers, it does not saturate for large earthquakes. Therefore, gravity signals are expected to provide a more robust early magnitude estimation than conventional seismic signals (Juhel et al., 2018).

Prompt elasto-gravity signals preceding seismic waves by 10 100 seconds have been detected on data recorded by superconducting gravimeters and broadband seismometers during several large earthquakes, including the Tohoku-oki earthquake $\left(M_{w}=9.1\right)$ (Montagner et al., 2016; Vallée et al., 2017; Vallée \& Juhel, 2019). The observed signals showed good agreement with simulations (Vallée et al., 2017; Juhel et al., 2019). These results suggested 
the potential of gravity measurements for EEWS. However, the capability of conventional inertial sensors, such as gravimeters and seismometers, to detect prompt gravity signals of earthquakes with magnitudes less than about 8 is almost impossible within a few tens of seconds. In fact, due to the equivalence principle, gravity changes cannot be distinguished from ground acceleration, thus background seismic noise severely challenges the detection of small-amplitude gravity signals. Moreover, gravity signals are partially canceled by the ground accelerations they induce, especially during the initial portion of the signal (Heaton, 2017; Vallée et al., 2017). In contrast, gravity gradiometry is not hampered by these fundamental limitations, as demonstrated in the context of gravitational wave detection (Harms, 2015). Therefore, gravity gradient measurements are necessary to detect earthquakes over the range of magnitudes and time scales that are critical for EEWS (Harms et al., 2015; Juhel et al., 2018).

For the purpose of earthquake detection, it is important to measure gravity gradient perturbations at frequencies around 0.1 $\mathrm{Hz}$, which corresponds to the typical timescale of moderate-tolarge earthquakes (few tens of seconds) and early warning $(\sim 10$ s). Several concepts of gravity gradiometers have been proposed to measure gravity gradients at such frequencies, originally in the context of low-frequency gravitational wave observation. Each detector is sensitive to different components of the gravity gradient tensor, thus their capability to detect earthquakes may be different. This is an essential consideration for the design of an actual gravity-based EEWS. Additionally, the spatial distribution of the signal amplitude is expected to depend on which components are measured, which should affect the optimal arrangement of a network of gravity-gradient detectors for EEWS.

The detectability of earthquakes of $M_{w}>7$ with gravity gradiometers was assessed via simulations by Juhel et al. (2018), who found that the sensitivity required for prompt detections is about $10^{-15} / \sqrt{\mathrm{Hz}}$ at $0.1 \mathrm{~Hz}$. That study was limited to the two verticalhorizontal gravity gradient components, $z x$ and $z y$. Here, we generalize these calculations to the gravity gradient signal induced by earthquakes for all components of the gravity gradient tensor. Based on the result, we compare the detectability of prompt gravity signals with different types of gravity gradiometers. We then analyze its dependence on the azimuth between the detector location and the earthquake epicenter, to determine the most favorable positions to install the detectors. The assumptions of the simulations are explained in Sec. 2. The simulation results for each component are presented in Sec. 3. The differences of detectability between the three gradiometer types are analyzed in Sec. 4 . The results are discussed in Sec. 5.

\section{SIMULATION ASSUMPTIONS}

\subsection{Medium and source models}

The gravity perturbation signal generated by a point source is calculated as the convolution of the Green's function and the source time function. To evaluate the Green's function, we assume a homogeneous half-space. The speed of P-wave and S-wave are set to $7.8 \mathrm{~km} / \mathrm{s}$ and $4.4 \mathrm{~km} / \mathrm{s}$, respectively, as in Juhel et al. (2018). We assume a buried point double-couple source with prescribed focal mechanism.

The fundamental solution for the gravity-gradient perturbation in this half-space model was derived in Harms (2016). The model is intentionally simplified: it ignores complexities such as the layering of Earth's materials, the finite size of earthquake sources,

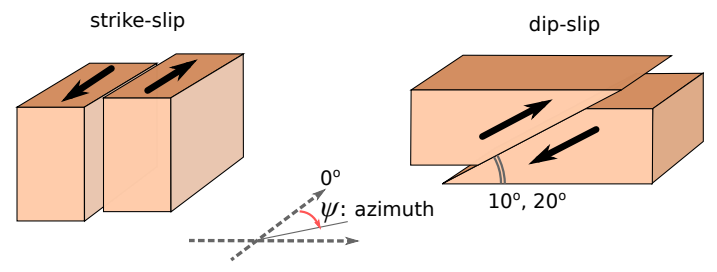

Figure 1. Earthquake focal mechanisms investigated in this paper, and definition of the azimuth $\Psi$.

non-double-couple components, and self-gravitation. Such ingredients can be taken into account by more computationally demanding methods, such as the normal mode theory (Juhel et al., 2019). However, they only have second-order effects on the gravity gradient within the first few tens of seconds relevant for EEWS (Harms, 2016). Thus, the simplified model adopted here is expected to provide first-order insight on the detectability of gravity perturbations.

We adopt the source time function model introduced by Juhel et al. (2018):

$$
\dot{M}(t)= \begin{cases}a \frac{M_{0}}{T}(t / T)^{2} & (0<t<T) \\ a \frac{M_{0}}{T}\left(1-(t / T-1)^{2}\right)^{6} & (T<t<2 T)\end{cases}
$$

where $T$ is the half-duration of the rupture, and $a \sim 1.48$ is a coefficient designed to satisfy $\int_{0}^{2 T} \dot{M}_{0} d t=M_{0}$. The rupture duration is taken from the empirical relation $2 T=\left(M_{0} / 10^{16} \mathrm{~N} \cdot \mathrm{m}\right)^{1 / 3}$ (Houston, 2001). Self-similarity of the fault rupture is assumed in this model. In particular, the initial evolution $(t<T)$ of the source time function is independent of the final magnitude. In the following calculations, the magnitude of the earthquake is set to $M_{w}=7.0$, hence $T=8 \mathrm{~s}$.

We investigate three representative focal mechanisms: a vertical strike-slip fault and reverse dip-slip faults with two dip angles (Fig. 1). Their strike, dip and rake angles are (strike, dip, rake) $=$ $\left(0^{\circ}, 90^{\circ}, 0^{\circ}\right),\left(180^{\circ}, 10^{\circ}, 90^{\circ}\right)$ and $\left(180^{\circ}, 20^{\circ}, 90^{\circ}\right)$, respectively. The hypocenter depth is set to $20 \mathrm{~km}$.

\subsection{Gravity gradient tensor}

The gravity gradient tensor is defined as

$$
\boldsymbol{\Gamma}(\boldsymbol{x}, t)=-\boldsymbol{\nabla} \otimes \boldsymbol{\nabla} \phi_{\mathrm{g}}(\boldsymbol{x}, t) .
$$

where $\phi_{\mathrm{g}}$ is the gravitational potential. The gravity strain tensor $\boldsymbol{h}(\boldsymbol{x}, t)$ is related to the gravity gradient by double time integration,

$$
\boldsymbol{h}(\boldsymbol{x}, t)=-\int_{0}^{t} d \tau \int_{0}^{\tau} d \tau^{\prime} \boldsymbol{\Gamma}\left(\boldsymbol{x}, \tau^{\prime}\right)
$$

Due to the symmetry $\Gamma_{i j}=\Gamma_{j i}$ and to Poisson's equation $\Delta \phi_{\mathrm{g}}=$ 0 yielding $\operatorname{Tr}(\boldsymbol{\Gamma})=0$, both $\boldsymbol{\Gamma}$ and $\boldsymbol{h}$ have only five independent components. Here, we set the five independent components of $\boldsymbol{h}$ as depicted in Fig. 2. They are based on the radial (r), tangential $(\mathrm{t})$ and vertical $(\mathrm{z})$ directions relative to the epicenter and detector positions. Two horizontal components, "plus" $(+)$ and "cross" $(\times)$, are defined as

$$
\left\{\begin{array}{l}
h_{+}=\left(h_{\mathrm{rr}}-h_{\mathrm{tt}}\right) / 2 \\
h_{\times}=h_{\mathrm{rt}}=h_{\mathrm{tr}}
\end{array}\right.
$$

These two components are the measurable degrees of freedom of horizontal detectors such as the torsion-bar. Their names (plus and cross) are derived from the polarization of gravitational waves. The other three independent components are chosen to be vertical: $h_{\mathrm{zz}}$, $h_{\mathrm{rz}}$ and $h_{\mathrm{tz}}$. 


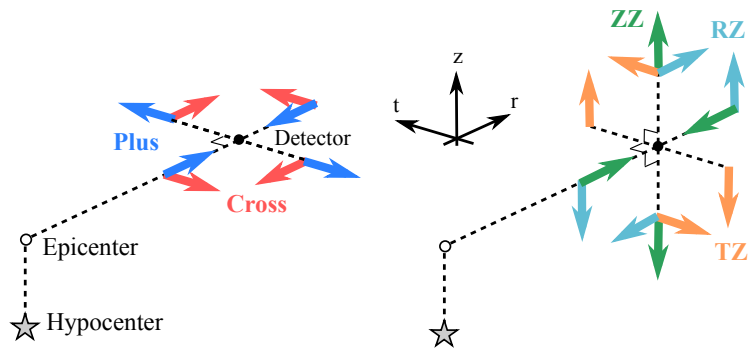

Figure 2. Definition of radial (r), transverse (t) and vertical (z) coordinates. The color arrows show the direction of the gravitational tidal forces for each component of the gravity strain tensor.

Each component of the gravity strain is approximated numerically as the finite difference of gravity perturbations between closely located observation points, integrated twice over time. We calculated gravity strain at various distances up to 200 or $1000 \mathrm{~km}$, and various azimuths ranging from $90^{\circ}$ to $270^{\circ}$. Here the azimuth angle $\psi$ is set to $0^{\circ}$ at the North and increases clockwise. Assuming symmetries, gravity strains at $\psi<90^{\circ}$ and at $\psi>270^{\circ}$ were inferred from the calculations at $90^{\circ}<\psi<270^{\circ}$ by considering $\boldsymbol{h}\left(\psi<90^{\circ}, \psi>270^{\circ}\right)=\boldsymbol{h}\left(\psi+180^{\circ}\right)$ for the strike-slip event and $\boldsymbol{h}\left(\psi<90^{\circ}, \psi>270^{\circ}\right)=\boldsymbol{h}\left(180^{\circ}-\psi\right)$ for the dip-slip events.

\subsection{Proposed gravity gradiometers}

Here we briefly present the three main types of low-frequency gravity gradiometers: superconducting gravity gradiometers, torsion bar antennas and atom interferometers.

A superconducting gravity gradiometer (SGG) measures differential motion between levitated masses induced by fluctuations of gravity gradient. The levitated masses behave like free-falling objects, so they are sensitive to the fluctuations at low frequencies. By combining the motions of several masses, all the components of the gravity gradient tensor can be measured. Such a configuration based on six levitated masses was proposed as the SOGRO concept (Superconducting Omni-directional Gravitational Radiation Observatory; Paik et al. (2016)).

A torsion-bar antenna measures the relative rotations of horizontally suspended bars induced by gravity gradient perturbations. The bars have low resonant frequency about horizontal rotations. A torsion bar is mainly sensitive to the horizontal components of the gravity gradient, $h_{+}$and $h_{\times}$. Although it can measure $h_{\mathrm{rz}}$ and $h_{\mathrm{tz}}$ via the vertical rotations (multi-output configuration; Shoda et al. (2017)), we do not consider that possibility here because its sensitivity has not been well discussed yet. Two configurations, TOBA (TOrsion-Bar Antenna; Ando et al. (2010)) and TorPeDO (Torsion Pendulum Dual Oscillator; McManus et al. (2017)), have been proposed. Although a single detector with two bars is sensitive to only one component, $h_{+}, h_{\times}$or a linear combination of them, both of the horizontal components can be measured by using two detectors with different azimuths.

An atom interferometer $(\mathrm{AI})$ measures gravity field perturbations via free-falling atoms (Keith et al., 1991). It uses matter waves to probe the gravitational field. The components of gravity gradient measurable with AI depend on the configuration. Original configurations of AI measure vertical gravitational acceleration which directly affects the atoms, hence they are sensitive to vertical gravity gradient. The recently proposed high-sensitivity configuration
MIGA (Matter wave-laser based Interferometer Gravitation Antenna; Canuel et al. (2018)) measures the gravitational effect on horizontally propagating lasers via the atoms, hence it is sensitive to horizontal gravity gradients.

Prototypes of some of these detectors are currently under development. They have roughly common target sensitivities of about $10^{-15} / \sqrt{\mathrm{Hz}}$ at $0.1 \mathrm{~Hz}$, and worse at lower frequencies. This sensitivity target is adopted here to calculate the detectability of earthquakes by next-generation gravity-gradient instruments.

\subsection{Detection signal-to-noise ratio}

Following Juhel et al. (2018), we consider an optimal matchedfilter detection procedure. The matched-filters are pre-whitened signal templates. The templates $h(\tau)$ are computed following the same procedure described in Section 2.1 for a complete set of earthquake locations, magnitudes and focal mechanisms. The whitened templates $h_{\mathrm{w}}(\tau)$ satisfy

$$
h_{\mathrm{w}}(\tau) \equiv \int_{-\infty}^{\infty} \frac{\tilde{h}(f)}{\sqrt{S_{\mathrm{n}}(f)}} e^{2 \pi i f \tau} d f
$$

where $\tilde{h}(f)$ is the Fourier-transform of $h$ and $\sqrt{S_{\mathrm{n}}(f)}$ is the amplitude spectral density (ASD) of detector noise. The matched-filter output is obtained by correlating the whitened template with the whitened data. The signal-to-noise ratio (SNR) is defined as the ratio between the matched-filter output and the standard deviation of the matched-filter applied to noise alone. The SNR value is denoted $\rho$ hereafter. A detection is declared if $\rho$ exceeds a certain threshold $\rho_{0}$. Under the assumption of Gaussian detector noise, the false-alarm probability is $\operatorname{erfc}\left(\rho_{0}\right)$ and the detection probability is $\operatorname{erfc}\left(\rho_{0}-\rho\right)$, where the complementary error function is defined as $\operatorname{erfc}(x)=\frac{1}{\sqrt{2 \pi}} \int_{x}^{\infty} e^{-t^{2} / 2} d t$ (Eqs. (1.11) and (1.12), respectively, from Chapter IV of Helstrom (1968)). Here, we choose $\rho_{0}=5$ as the threshold SNR, which corresponds to a false-alarm probability of $3 \times 10^{-7}$.

The following ASD of detector noise is assumed, as in Juhel et al. (2018):

$$
\sqrt{S_{\mathrm{n}}(f)}=\frac{(0.1 \mathrm{~Hz})^{2}+f^{2}}{f^{2}} 10^{-15} / \sqrt{\mathrm{Hz}} .
$$

The floor noise level of $10^{-15} / \sqrt{\mathrm{Hz}}$ and the cutoff frequency of $0.1 \mathrm{~Hz}$ are close to the design noise levels of proposed gravity gradiometers (Shimoda et al., 2020). A case study of the actual design sensitivity of TOBA is given in Appendix A. Though the actual noise spectrum may differ in detail for each tensor component and for each type of detector, in the first-order approach taken here we assume a common noise level. For real-time application, whitening is implemented in time domain as a second-order high-pass filter, such as a Butterworth filter, with $0.1 \mathrm{~Hz}$ cutoff frequency, whose Fourier transform turns out to be $1 / \sqrt{S_{\mathrm{n}}(f)}$.

To evaluate the matched-filter detection performance we could draw many random realizations of noise, compute the SNR for each signal plus noise realization and average, as done by Juhel et al. (2018). A more efficient approach adopted here is to evaluate the following expression of the optimal SNR (Turin, 1960; Jaranowski \& Królak, 2012):

$$
\rho=\sqrt{2 \int_{-\infty}^{\infty} \frac{|\tilde{h}(f)|^{2}}{S_{\mathrm{n}}(f)} d f}=\sqrt{2 \int_{0}^{t}\left|h_{\mathrm{w}}(\tau)\right|^{2} d \tau}
$$

at the detection time $t$ (the origin of time is set at the rupture onset). This optimal SNR is achieved in the ideal case when the tem- 
plate used for matched-filter detection is a perfect representation of the real signal. This idealization is not severely restrictive: since prompt gravity signals depend on a smoothed version of the source time function (e.g. in an unbounded space they are proportional to the second time-integral of the source time function (Harms et al., 2015)), their detection does not depend strongly on the detailed shape of the signal template (Juhel et al., 2018).

\section{SNR DISTRIBUTION FOR EACH GRAVITY GRADIENT COMPONENT}

Here we show the calculated SNR distribution for each component of the gravity strain tensor, calculated at two reference times: 10 seconds after the onset of the fault rupture and at the arrival time of $\mathrm{P}$-waves at each detector location. The former is essential for rapid detection in EEWS. Although detection before 10 seconds would be better, in Appendix B it is shown to be challenging with the proposed gravity gradiometers. The latter reference time is important for the robust estimation of earthquake source parameters such as final magnitude.

\subsection{SNR at 10 seconds after rupture onset}

Fig. 3 shows the SNR distributions at 10 seconds after rupture onset, at epicentral distances up to $200 \mathrm{~km}$, for the three focal mechanism and the five gravity strain components. We define a "highSNR region" as the region where SNR $>5$ (inside the dashed contour), in which we can expect a reliable detection with a single detector.

The symmetry of the volumetric ground deformation produced by the different focal mechanisms, which controls the density perturbations, explains the azimuth distribution of SNR. The resulting pattern of SNR has four lobes for the strike-slip earthquake and two lobes for the dip-slip earthquakes.

For the strike-slip earthquake (left column of Fig. 3), the gravity gradient perturbation is largest in the two horizontal components, plus and cross. Their high-SNR region extends up to about $140 \mathrm{~km}$ from the epicenter. Only the cross component has high SNR at azimuths $0^{\circ}, 90^{\circ}, 180^{\circ}$ and $270^{\circ}$. The $\mathrm{ZZ}$ and RZ components are also important; their SNRs are about $50 \%$ and $70 \%$ of the plus component, respectively. Though the TZ component has the lowest SNR, in particular much lower than the cross component, it is the only vertical component which can detect the earthquake at $\psi=0^{\circ}, 90^{\circ}, 180^{\circ}$ and $270^{\circ}$.

For the dip-slip events, the vertical components are as important as the horizontal components. The $\mathrm{ZZ}$ component is dominant at $270^{\circ}$ and the $\mathrm{RZ}$ component is largest at $90^{\circ}$, whose high-SNR region reaches over $135 \mathrm{~km}$. Among the horizontal components, the plus component has almost the same SNR $(\sim 95 \%)$ as the $\mathrm{ZZ}$ component at $270^{\circ}$. At the along-strike azimuths $\left(0^{\circ}\right.$ and $\left.180^{\circ}\right)$, all the components have small SNR, because the ground is not initially compressed or dilated in these directions. Only the cross and the TZ component have detection capability in these azimuths.

As the dip angle of the dip-slip event increases from $10^{\circ}$ to $20^{\circ}$, the asymmetries of the SNR distributions between the east half and the west half tend to be larger. The SNRs increase by 5-20 $\%$ in the azimuths of largest high-SNR region $\left(270^{\circ}\right.$ for the plus and the $\mathrm{ZZ}$ components, $90^{\circ}$ for the $\mathrm{RZ}$ component), and decrease significantly in the opposite directions.

\subsection{SNR at P-wave arrival time}

Fig. 4 shows the SNR distribution at P-wave arrival time up to $1000 \mathrm{~km}$ distance. In the region with SNR $>100$ (inside the dotted line) the signals can be measured very precisely and the earthquake source parameters can be estimated reliably. The general trends of the SNR distributions are similar to those at 10 seconds.

For the strike-slip event, at least two components provide good SNRs at each azimuth. The detectable region $(\mathrm{SNR}>5$ ) extends over more than $1000 \mathrm{~km}$ at all azimuths. The horizontal components have the highest SNR within $350 \mathrm{~km}$ to the epicenter, while the SNR of the RZ component is highest beyond $350 \mathrm{~km}$ distance at $45^{\circ}, 135^{\circ}, 225^{\circ}$ and $315^{\circ}$.

For the dip-slip events, the plus, ZZ and RZ components contribute to the detection in the along-dip directions (around $90^{\circ}$ and $270^{\circ}$ ). However, the appropriate range for parameter estimations $(\mathrm{SNR}>100)$ is narrower than for the strike-slip event, while the appropriate range for detection (SNR $>5$ ) at 10 seconds is almost the same in the optimal directions for both type of events (Fig. 3). As the dip angle increases, the distance with SNR $>100$ increases at all directions except at distances $<250 \mathrm{~km}$. In the along-strike directions $\left(0^{\circ}\right.$ and $\left.180^{\circ}\right)$, only the plus component has a wide detectable range.

\section{SUM OF SNR FOR DIFFERENT SETS OF GRAVITY STRAIN COMPONENTS}

Here, in order to assess which of the three kinds of gravity gradiometers described in Sec. 2.3 is preferred, we separate the horizontal set (plus and cross) and the vertical set (ZZ, RZ and TZ) of components because some of the gradiometers are sensitive to only one of them, while a superconducting gravity gradiometer like SOGRO can measure all components. The total SNR of all components, all horizontal components and all vertical components are calculated by taking the square root of the sum of squares of SNRs of individual components, based on Jaranowski \& Królak (2012). The detector noises in the measurements of the components are assumed to be uncorrelated to each other. Although all the results shown in this section are the direct consequence of the previous section (Sec. 3), they are presented here to enable easy comparison between the types of gradiometers.

\subsection{SNR at 10 seconds after the onset of the fault rupture}

The total SNRs at 10 seconds after rupture onset are shown in Fig. 5. Fig. 6 shows the azimuth distribution of the SNR at $100 \mathrm{~km}$ distance from the epicenter.

The total detectable range at $10 \mathrm{~s}$ for the strike-slip event is roughly the same at all azimuths. It is about $140-150 \mathrm{~km}$ for all components and for horizontal-only components. This is because the plus and the cross components are dominant for this event as shown in Sec. 3. With the vertical components, the detectable distance is $135 \mathrm{~km}$ at $45^{\circ}, 135^{\circ}, 225^{\circ}$ and $315^{\circ}$, and smaller than 110 $\mathrm{km}$ at $0^{\circ}, 90^{\circ}, 180^{\circ}$ and $270^{\circ}$. Compared with the horizontal components, the SNR of the vertical components is about $80 \%$ at the optimal directions, while it is less than $40 \%$ at the least favorable directions (Fig. 6).

For dip-slip events, the detectable distance with all components has minima in the along-strike directions $\left(0^{\circ}\right.$ and $\left.180^{\circ}\right)$, because no component has very good SNR there, though the detection before the $\mathrm{P}$-wave arrival is possible at every direction. The 

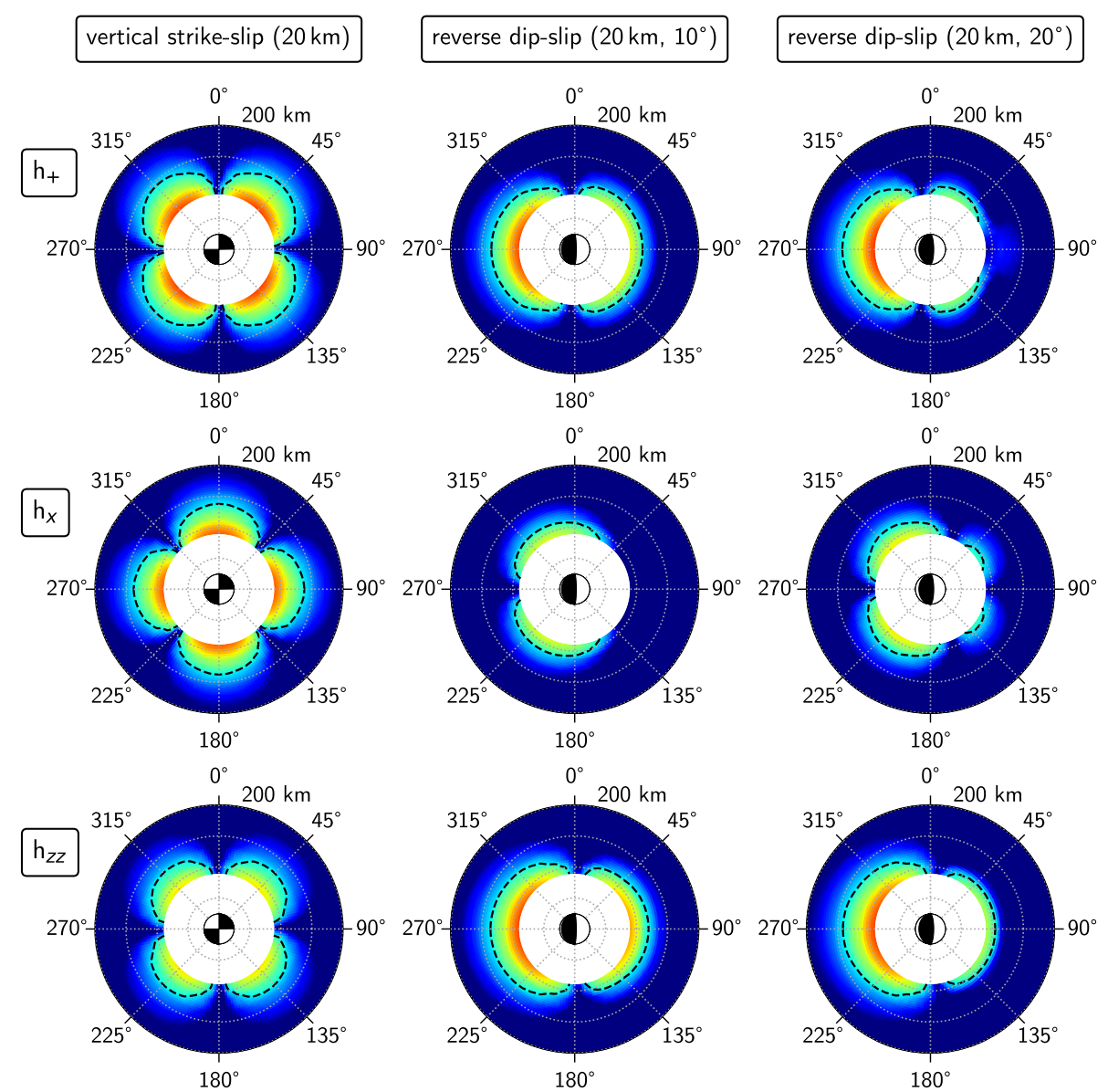

$0^{\circ}$
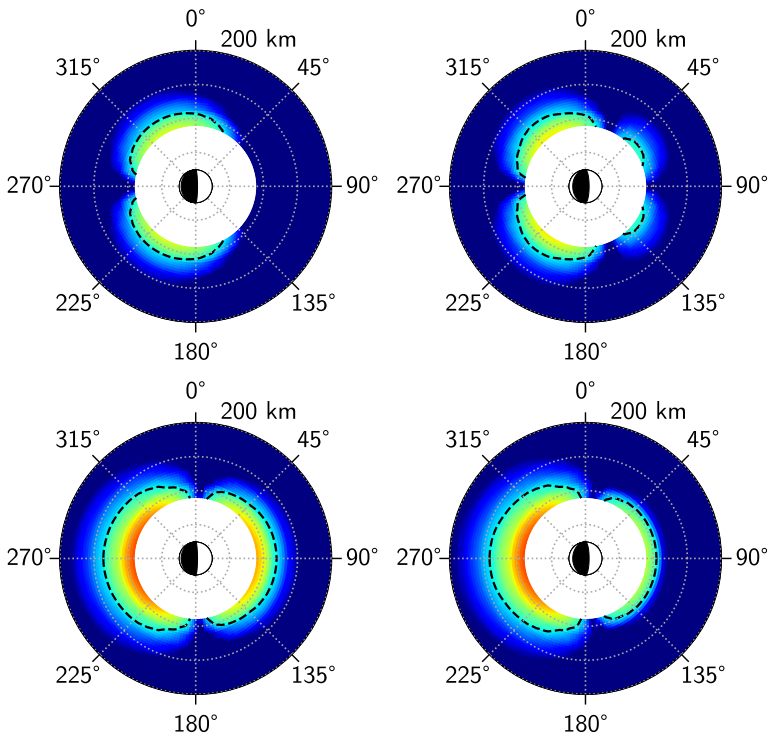

$180^{\circ}$

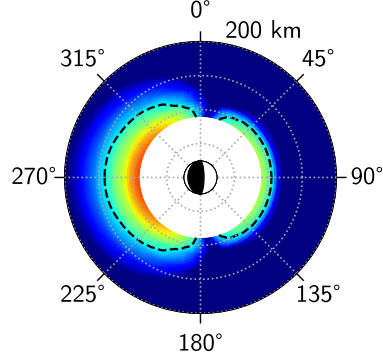

$0^{\circ}$

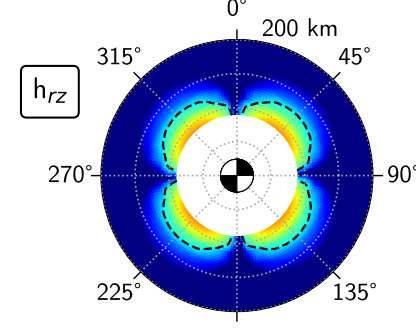

$0^{\circ}$
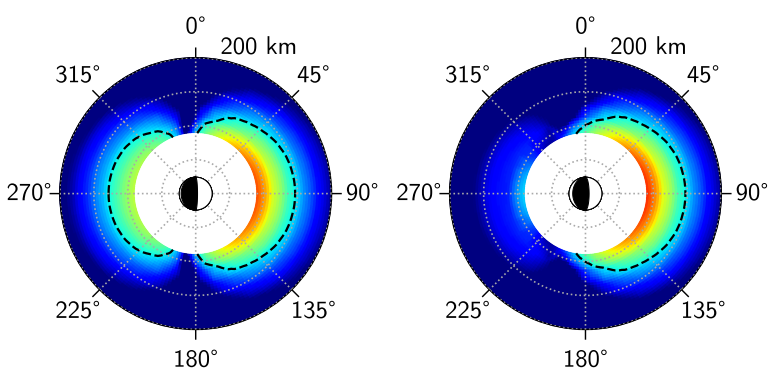

$180^{\circ}$

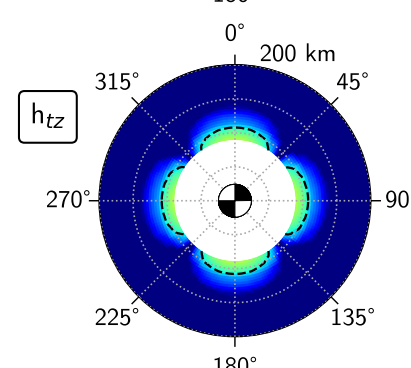

$0^{\circ}$

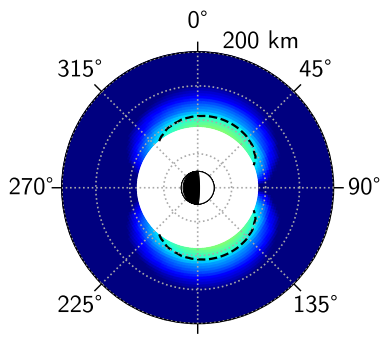

$180^{\circ}$

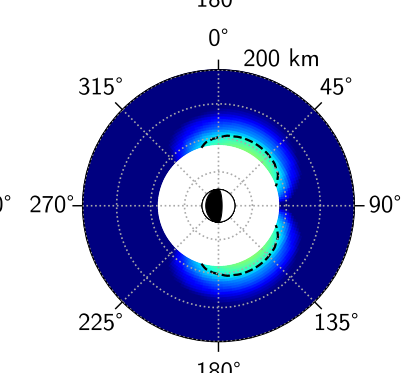

$180^{\circ}$

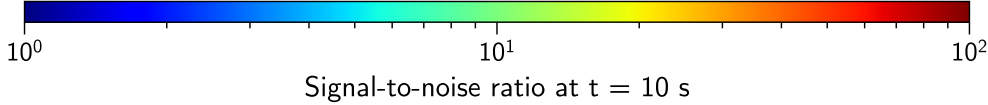

Figure 3. Azimuth-distance distributions of the SNR of matched-filter earthquake detection with gravity strain measurements at 10 seconds after rupture onset. The detector noise level is set to $10^{-15} / \sqrt{\mathrm{Hz}}$ at $0.1 \mathrm{~Hz}$. The magnitude is $M_{w}=7.0$. Colors indicate the SNR according to the color bar shown at the bottom. Each column corresponds to one of the three focal mechanisms defined in Fig. 1, and each row to one of the five components of gravity gradient tensor defined in Fig. 2. The dotted grey circles indicate distances of 50, 100 and $150 \mathrm{~km}$. The dashed black curve is the contour SNR $=5$. The white blank area $(\lesssim 80 \mathrm{~km})$ is the region where seismic P-waves arrive within 10 seconds after rupture onset. 


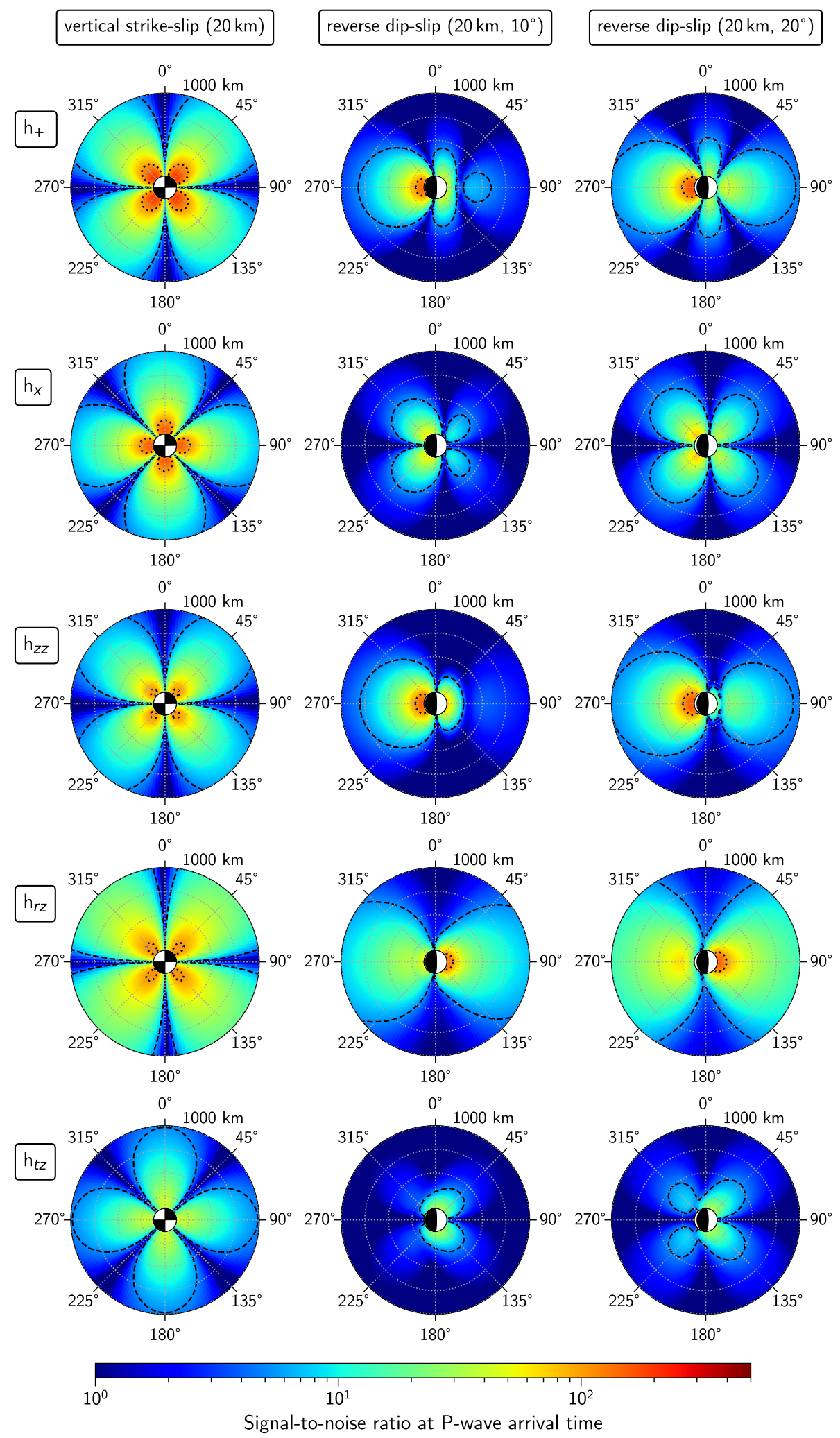

Figure 4. Same as Fig. 3 but gravity strain measurements at the P-wave arrival time and up to an epicentral distance of $1000 \mathrm{~km}$. The magnitude is $M_{w}=7.0$. The dotted grey circles indicate distances of 250,500 and $750 \mathrm{~km}$. The dashed and dotted lines are contours for SNR $=5$ and 100 , respectively. 


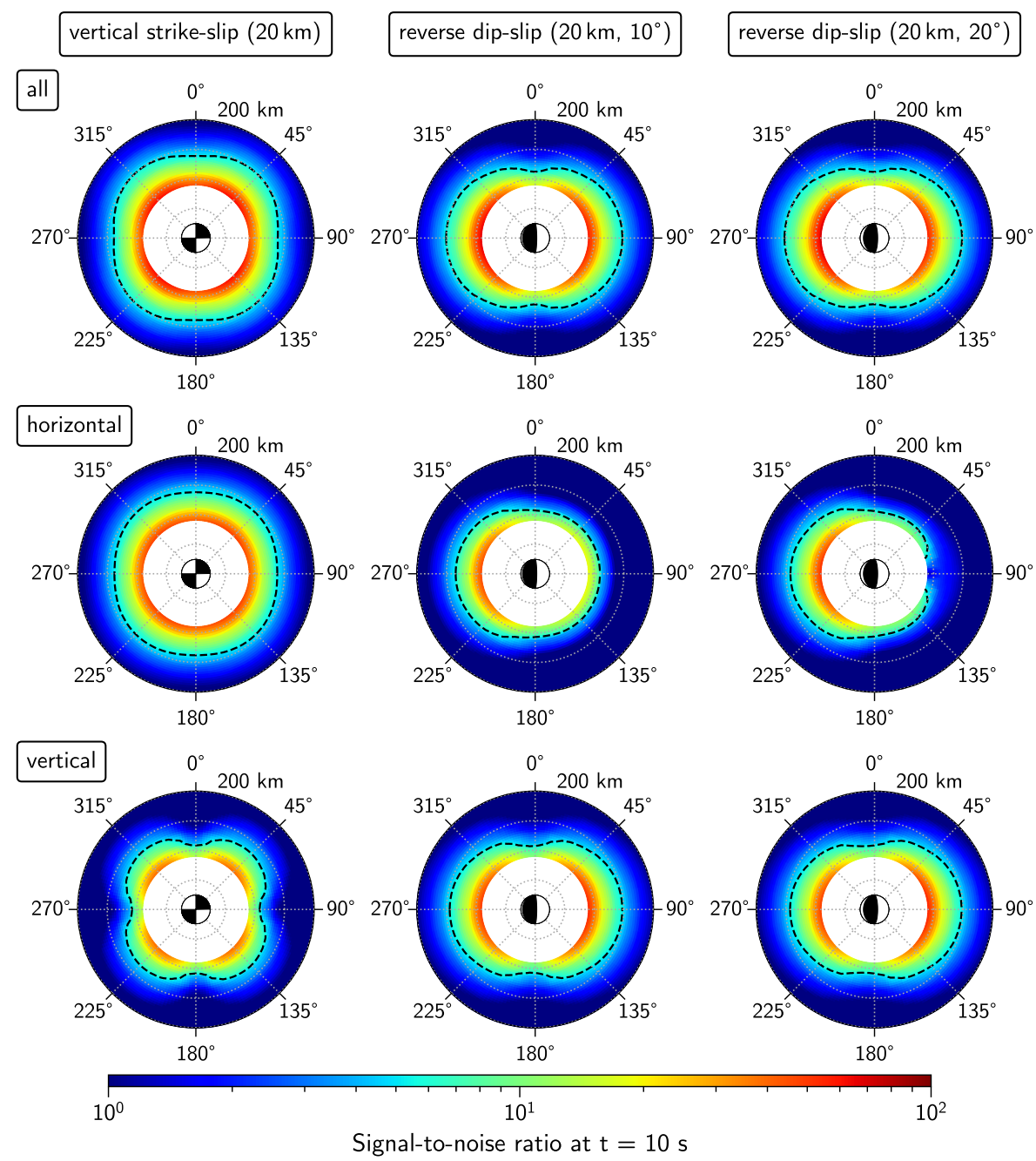

Figure 5. Total SNR for the three types of detectors at 10 seconds after rupture onset, up to $200 \mathrm{~km}$ distance. Each row corresponds to a different set of components of gravity strain: all (top), only horizontal (middle) and only vertical (bottom). The magnitude is $M_{w}=7.0$.
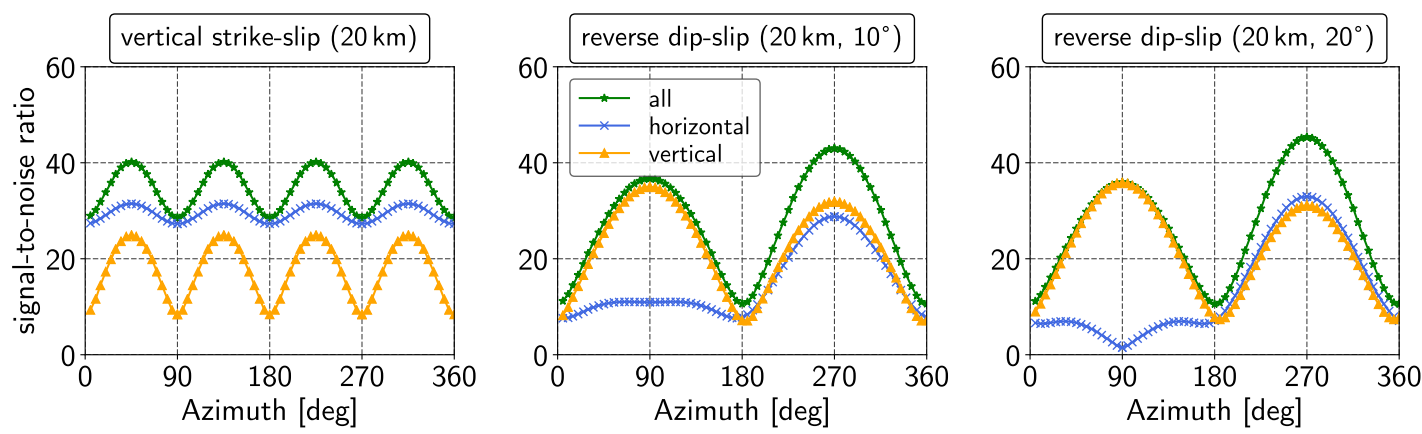

Figure 6. Azimuth distribution of total SNR at 10 seconds after rupture onset, at $100 \mathrm{~km}$ distance. The magnitude is $M_{w}=7.0$.

detectable distance for the event with $10^{\circ}$ dip using horizontal components only is about $135 \mathrm{~km}$ around $270^{\circ}$. The set of vertical components provides a better detectable distance of $145 \mathrm{~km}$ at $270^{\circ}$, and $150 \mathrm{~km}$ at $90^{\circ}$. As the dip angle increases from $10^{\circ}$ to $20^{\circ}$, the detectable distance with the horizontal components slightly grows to $140 \mathrm{~km}$ at $270^{\circ}$, which is almost the same as that with the vertical components. The SNR of the horizontal components is similar to that of the vertical components at $270^{\circ}$, but much less at $90^{\circ}$ (Fig. 6). 


\subsection{SNR at P-wave arrival time}

The total SNRs at P-wave arrival time are shown in Fig. 7. Fig. 8 shows the SNR at $300 \mathrm{~km}$ distance from the epicenter.

For the strike-slip event, the observable range with the horizontal components is almost isotropic, while the vertical components can observe earthquakes in more limited directions. For dipslip earthquakes, the detectability strongly depends on azimuth for any sets of strain components, and the SNR is higher in the half area around $270^{\circ}$ direction. Especially the SNR of the horizontal components is small at $90^{\circ}$. The observable range of the dip-slip earthquakes is narrower than the strike-slip event, but it becomes wider as the dip angle increases.

\section{DISCUSSION}

For strike-slip earthquakes, the results in Secs. 3 and 4 show that the horizontal components of the gravity strain perturbation are essential for rapid detection. Hence the measurement of the horizontal gravity strain, using torsion-bar detectors or atom interferometers like MIGA, seems sufficient for EEWS. For such detectors, there is no preferred direction in which the detectors should be placed, since the total SNR distribution of the strike-slip earthquake using both horizontal components is almost isotropic. Additional observation of the vertical gravity strain slightly improves the detectable distance from $145 \mathrm{~km}$ to $150 \mathrm{~km}$ at $\psi=45^{\circ}, 135^{\circ}, 225^{\circ}$ and $315^{\circ}$, but has less benefit in the other directions. The vertical gravity strain has an advantage only for source parameter estimation at the P-wave arrival time, especially at the distant locations ( $>350$ $\mathrm{km})$.

For dip-slip subduction thrust earthquakes, the strike is usually parallel to the coastline and detectors can only be installed on land. This corresponds to the $180^{\circ}-360^{\circ}$ azimuth range here, in which the horizontal and the vertical gravity strain have similar SNR (Figs. 3 - 8). Although prompt detection is achievable with only the horizontal gravity strain up to $135 \mathrm{~km}$ at $270^{\circ}$, additional observation of the vertical gravity strain can improve the detectable distance to $150 \mathrm{~km}$. Therefore, torsion-bar detectors or atom interferometers seem sufficient for subduction earthquake detection, while superconducting gravity gradiometers like SOGRO can improve SNR by a factor $\sim \sqrt{2}$. The same statement is applicable to the observation at the P-wave arrival time.

As earthquake focal mechanisms varies from place to place, the preferred type of the detector is not globally unique. Although measuring all strain components with SOGRO-like detectors can be always expected to improve earthquake detection, the information presented above can be useful to determine which aspects of the improvements are actually cost-effective in each region.

The discussions above are mainly focused on the detectability of earthquakes by template-matching. However, there are additional considerations for epicenter localization. It has been proposed that the ratio of signal amplitude between two horizontal gravity gradient components is useful to identify the source location (McManus et al., 2018). That approach can be applied more generally to the five components, which should improve the location accuracy. Therefore, observing all components of gravity strain may be important for event location, even if some do not have high SNR.

We adopted simplifying assumptions in our calculations, such as the source time function model (Eq. (1)) and the homogeneous half-space. For actual earthquakes, the SNR can be different than in our calculations due to the diversity of the source time functions, especially their onsets (Meier et al., 2017). Future work is required to address how the heterogeneities of the Earth structure may affect the gravity strain signals.

\section{CONCLUSION}

The detectability of earthquakes using different kinds of gravity gradiometers has been quantified and discussed, for strike-slip and dip-slip faults, in order to investigate what kind of detectors are preferred. The calculated SNR distributions show that the horizontal strain components are essential for the detection of strike-slip earthquakes, and the horizontal and the vertical strain components have similar SNR for dip-slip earthquakes. Therefore, torsion-bar detectors like TOBA or atom interferometer like MIGA are sufficient for earthquake early warning applications. The detectable range at 10 seconds after rupture onset with horizontal gravity strains is $140 \mathrm{~km}$ for strike-slip earthquakes and $135 \mathrm{~km}$ for dip-slip earthquakes. A superconducting gravity gradiometer which can measure both the horizontal and the vertical gravity strains has higher SNR by roughly $\sqrt{2}$ times for dip-slip earthquakes, while the benefit of the vertical gravity strain is less for strike-slip earthquakes. Hence, SOGRO is a better choice only if it is available with less technical difficulties than TOBA or MIGA. However, if the measurement of vertical gravity strain with SOGRO is technically difficult, it is better to use two horizontal detectors instead. Future experimental work is necessary to determine the optimal choice of the detectors.

Although actual earthquakes are not as simple as modeled in this paper, our results provide insight on the fundamental differences between the different proposed types of gravity gradiometers. Such information is useful for the design of a gravity-based earthquake early warning system.

\section{ACKNOWLEDGMENTS}

We acknowledge the financial support from the UnivEarthS Labex program at Sorbonne Paris Cité (ANR-10-LABX-0023 and ANR11-IDEX-0005-02) and the financial support of the Agence Nationale de la Recherche (ANR) through the grant ANR-14-CE030014-01. This study contributes to the IdEx Université de Paris ANR-18-IDEX-0001. T. S. acknowledges the financial support of GRASP (Graduate Research Abroad in Science Program) managed by University of Tokyo. J.-P. M. acknowledges the financial support of Institut Universitaire de France. J. P. A. acknowledges funding by the French government through the "Investissements d'Avenir UCAJEDI" project managed by the ANR through grant ANR-15IDEX-01. We are grateful to Jan Harms for sharing his code to compute gravity changes. Numerical computations were performed on the S-CAPAD platform, IPGP, France. Python routines used to compute the expected gravity strain signal (Harms, 2016) and the optimal signal-to-noise ratio are available at the GitHub repository https://github.com/kjuhel/gravity-eew.

\section{References}

Allen, R. M. \& Melgar, D., 2019. Earthquake early warning: Advances, scientific challenges, and societal needs, Annual Review of Earth and Planetary Sciences, 47(1), 361-388. 

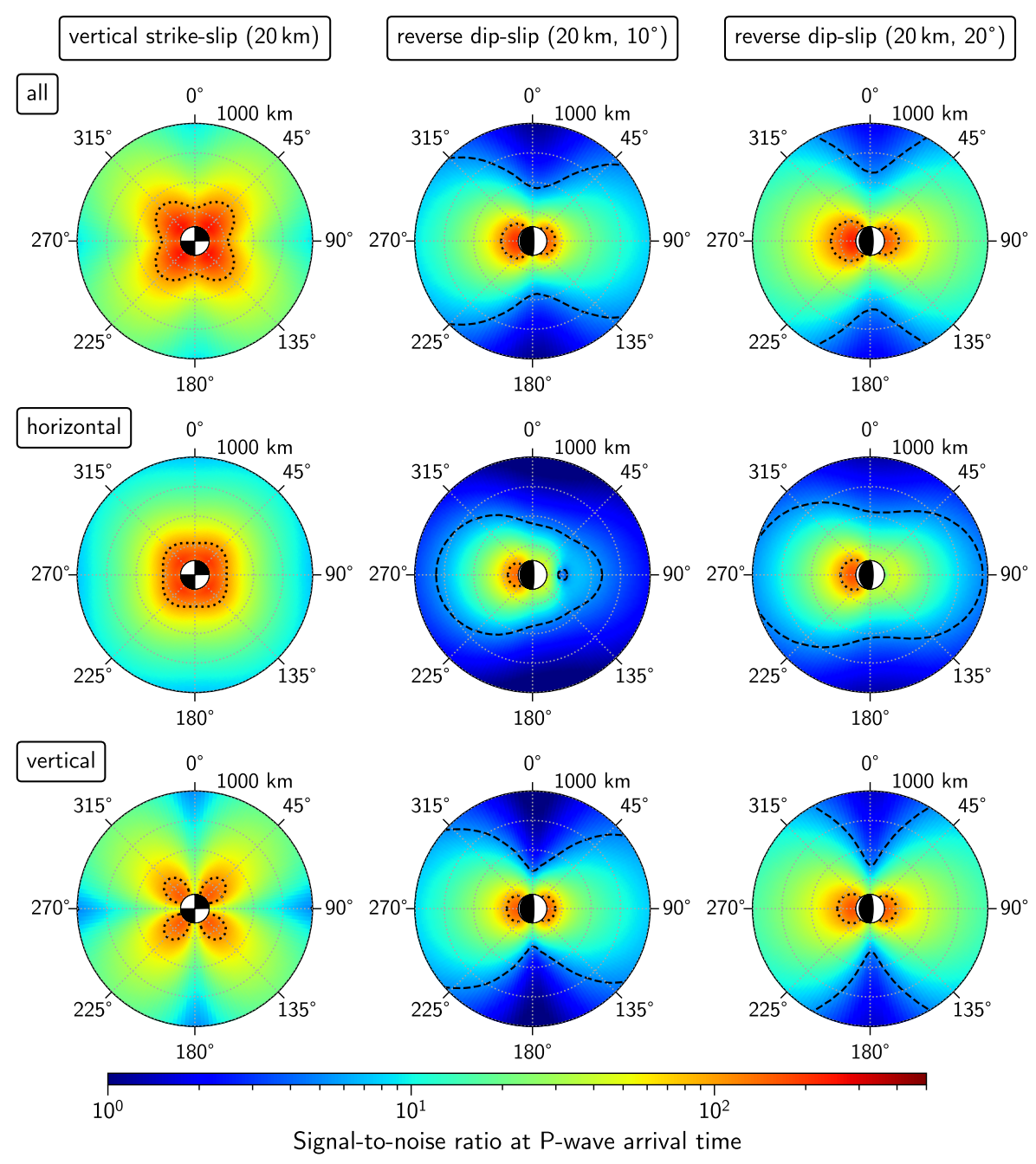

Figure 7. Total SNR for the three types of detectors at P-wave arrival time, up to $1000 \mathrm{~km}$ distance.
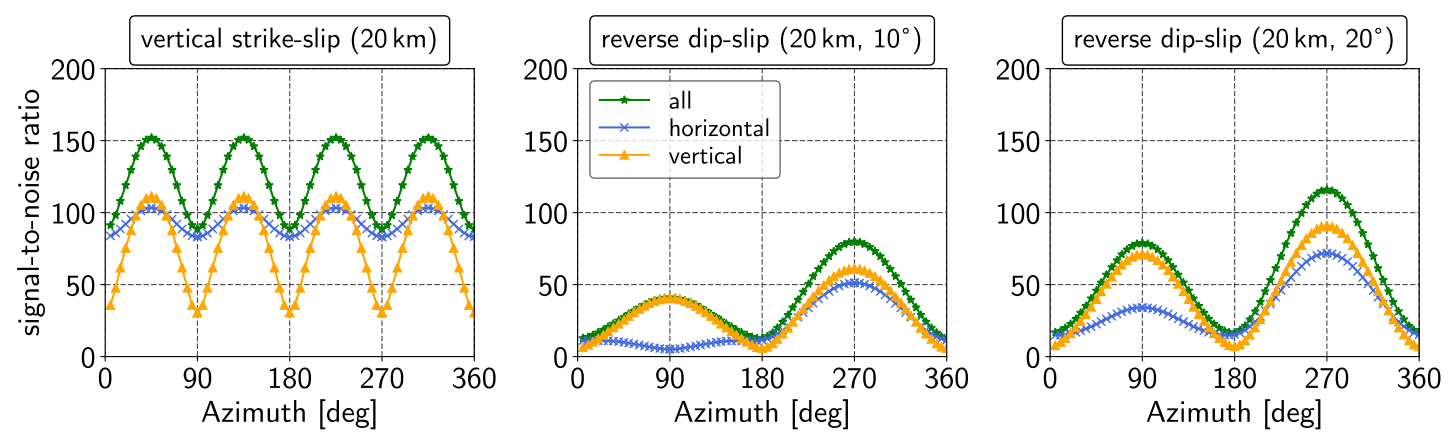

Figure 8. Azimuth distribution of total SNR at P-wave arrival time, at $300 \mathrm{~km}$ distance.

Ando, M., Ishidoshiro, K., Yamamoto, K., Yagi, K., Kokuyama, W., Tsubono, K., \& Takamori, A., 2010. Torsion-Bar Antenna for Low-Frequency Gravitational-Wave Observations, Phys. Rev. Lett., 105, 161101.

Canuel, B., Bertoldi, A., Amand, L., Pozzo di Borgo, E., Chantrait, T., Danquigny, C., Dovale Álvarez, M., Fang, B.,
Freise, A., Geiger, R., Gillot, J., Henry, S., Hinderer, J., Holleville, D., Junca, J., Lefèvre, G., Merzougui, M., Mielec, N., Monfret, T., Pelisson, S., Prevedelli, M., Reynaud, S., Riou, I., Rogister, Y., Rosat, S., Cormier, E., Landragin, A., Chaibi, W., Gaffet, S., \& Bouyer, P., 2018. Exploring gravity with the MIGA large scale atom interferometer, Scientific Reports, 8(1), 14064. 
Harms, J., 2015. Terrestrial gravity fluctuations, Living Reviews in Relativity, 18(1), 3.

Harms, J., 2016. Transient gravity perturbations from a doublecouple in a homogeneous half-space, Geophysical Journal International, 205(2), 1153-1164.

Harms, J., Ampuero, J.-P., Barsuglia, M., Chassande-Mottin, E., Montagner, J.-P., Somala, S. N., \& Whiting, B. F., 2015. Transient gravity perturbations induced by earthquake rupture, Geophysical Journal International, 201(3), 1416-1425.

Heaton, T. H., 2017. Correspondence: Response of a gravimeter to an instantaneous step in gravity, Nature Communications, 8(1), 966.

Helstrom, C. W., 1968. Statistical Theory of Signal Detection 2nd Edition, Pergamon Press.

Houston, H., 2001. Influence of depth, focal mechanism, and tectonic setting on the shape and duration of earthquake source time functions, Journal of Geophysical Research: Solid Earth, 106(B6), 11137-11150.

Jaranowski, P. \& Królak, A., 2012. Gravitational-wave data analysis. formalism and sample applications: The gaussian case, Living Reviews in Relativity, 15(1), 4.

Juhel, K., Ampuero, J. P., Barsuglia, M., Bernard, P., ChassandeMottin, E., Fiorucci, D., Harms, J., Montagner, J.-P., Vallée, M., \& Whiting, B. F., 2018. Earthquake Early Warning Using Future Generation Gravity Strainmeters, Journal of Geophysical Research: Solid Earth, 123(12), 10,889-10,902.

Juhel, K., Montagner, J.-P., Vallée, M., Ampuero, J. P., Barsuglia, M., Bernard, P., Clévédé, E., Harms, J., \& Whiting, B. F., 2019. Normal mode simulation of prompt elastogravity signals induced by an earthquake rupture, Geophysical Journal International, 216(2), 935-947.

Keith, D. W., Ekstrom, C. R., Turchette, Q. A., \& Pritchard, D. E., 1991. An interferometer for atoms, Phys. Rev. Lett., 66, 26932696.

McManus, D. J., Forsyth, P. W. F., Yap, M. J., Ward, R. L., Shaddock, D. A., McClelland, D. E., \& Slagmolen, B. J. J., 2017. Mechanical characterisation of the TorPeDO: a low frequency gravitational force sensor, Classical and Quantum Gravity, 34(13), 135002.

McManus, D. J., Forsyth, P. W. F., Holland, N. A., Ward, R. L., Shaddock, D. A., McClelland, D. E., \& Slagmolen, B. J. J., 2018. Early Earthquake Detection with a Dual Torsion-Beam Gravimeter, arXiv, 1809.04787.

Meier, M.-A., Ampuero, J. P., \& Heaton, T. H., 2017. The hidden simplicity of subduction megathrust earthquakes, Science, 357(6357), 1277-1281.

Montagner, J.-P., Juhel, K., Barsuglia, M., Ampuero, J. P., Chassande-Mottin, E., Harms, J., Whiting, B., Bernard, P., Clévédé, E., \& Lognonné, P., 2016. Prompt gravity signal induced by the 2011 Tohoku-Oki earthquake, Nature Communications, 7, $13349 \mathrm{EP}$-.

Paik, H. J., Griggs, C. E., Moody, M. V., Venkateswara, K., Lee, H. M., Nielsen, A. B., Majorana, E., \& Harms, J., 2016. Lowfrequency terrestrial tensor gravitational-wave detector, Classical and Quantum Gravity, 33(7), 075003.

Shimoda, T., Takano, S., Ooi, C. P., Aritomi, N., Michimura, Y., Ando, M., \& Shoda, A., 2020. Torsion-bar antenna: A ground-based mid-frequency and low-frequency gravitational wave detector, International Journal of Modern Physics D, 29, 04, 1940003.

Shoda, A., Kuwahara, Y., Ando, M., Eda, K., Tejima, K., Aso, Y., \& Itoh, Y., 2017. Ground-based low-frequency gravitational-

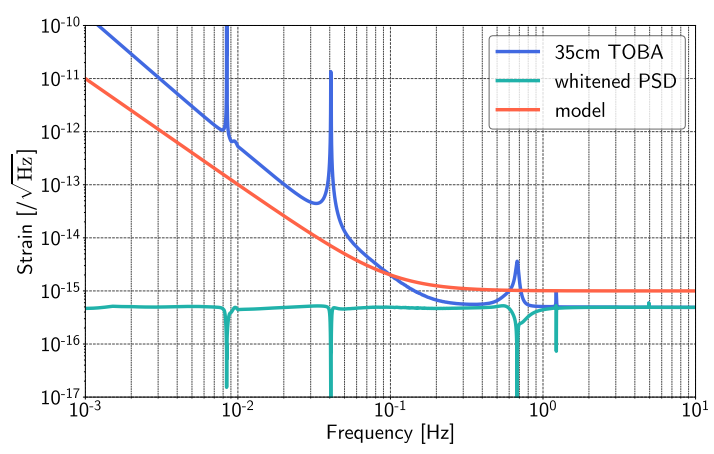

Figure 9. Design sensitivity of $35 \mathrm{~cm}$-scale torsion-bar antenna (Shimoda et al.(2020)). The amplitude spectral density of the detector noise in unit of gravity strain (blue line), and the whitened noise to calculate the SNR (green line). The model sensitivity (Eq. (6), red line) is also shown for comparison.

wave detector with multiple outputs, Phys. Rev. D, 95, 082004. Sladen, A., Rivet, D., Ampuero, J. P., De Barros, L., Hello, Y., Calbris, G., \& Lamare, P., 2019. Distributed sensing of earthquakes and ocean-solid earth interactions on seafloor telecom cables, Nature Communications, 10(1), 5777.

Turin, G., 1960. An introduction to matched filters, IRE Transactions on Information Theory, 6(3), 311-329.

Vallée, M. \& Juhel, K., 2019. Multiple observations of the prompt elastogravity signals heralding direct seismic waves, Journal of Geophysical Research: Solid Earth, 124(3), 2970-2989.

Vallée, M., Ampuero, J. P., Juhel, K., Bernard, P., Montagner, J.P., \& Barsuglia, M., 2017. Observations and modeling of the elastogravity signals preceding direct seismic waves, Science, 358(6367), 1164-1168.

\section{Appendix A. SNR with the design sensitivity of $35 \mathrm{~cm}$ TOBA}

As an example of an actual gravity gradiometer, a $35 \mathrm{~cm}$-scale torsion-bar antenna is investigated in this appendix. Fig. 9 shows the design sensitivity of the detector (Shimoda et al., 2020) (blue line) and the model sensitivity used in the main text (Eq. (6), red line). The differences from the model sensitivity are as follows: the design sensitivity is proportional to the $-2.5^{\text {th }}$ power of frequency below $0.1 \mathrm{~Hz}$, which is limited by the thermal fluctuation of the pendulum, and has peaks at $8.5 \mathrm{mHz}, 40 \mathrm{mHz}$ and $0.67 \mathrm{~Hz}$ due to the resonances of the pendulum. Accordingly, the whitening process for the calculation of SNR (Eq. (5)) is also changed to the combination of several filters for the low-frequency noise and the resonant peaks. The whitened noise spectrum is shown with the green line in Fig. 9.

The calculated SNRs at 10 seconds after the onset of the fault rupture are shown in Fig. 10. Each color map shows the sum of two horizontal gravity strain components for each type of earthquake. The prompt detection is achievable with the $35 \mathrm{~cm}$ TOBA up to about $130 \mathrm{~km}$ distance, though the SNRs are smaller by $40 \%$ than those with the model noise shown in Fig. 5. On the other hand, the SNRs at the P-wave arrival time, which are shown in Fig. 11, are much smaller than those of the model noise (Fig. 7); e.g. by a factor of 5 at $300 \mathrm{~km}$ distance for the strike-slip earthquake. As indicated in Juhel et al. (2018), the low-frequency sensitivity is important for the observation at the P-wave arrival time because the larger 


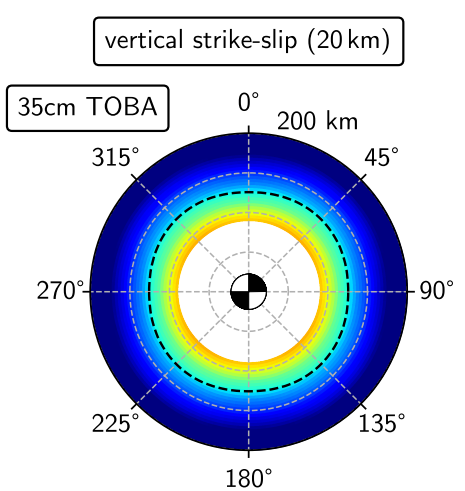

$180^{\circ}$

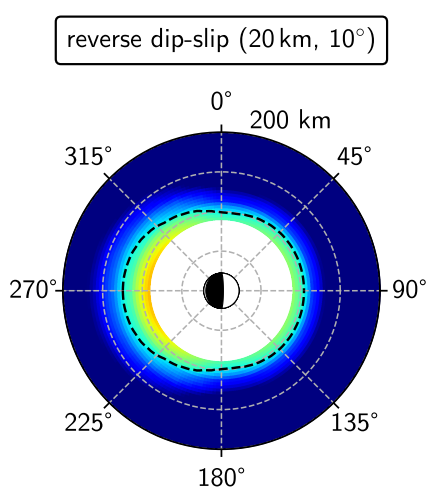

$180^{\circ}$

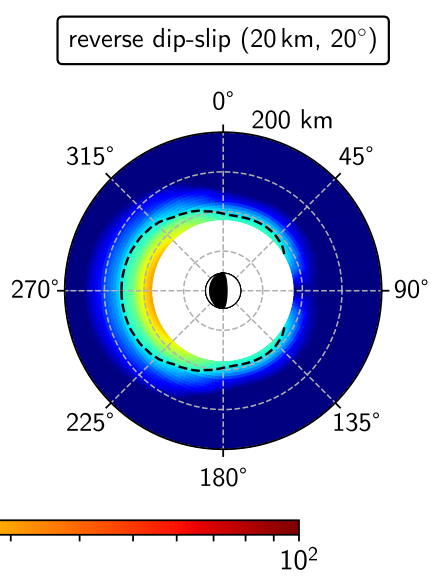

Signal-to-noise ratio at $\mathrm{t}=10 \mathrm{~s}$

Figure 10. Azimuth-distance distributions of SNR using $35 \mathrm{~cm}$-scale TOBA at 10 seconds after the onset of the fault rupture. Sum of the SNRs of two horizontal gravity strain components is shown in each color map.

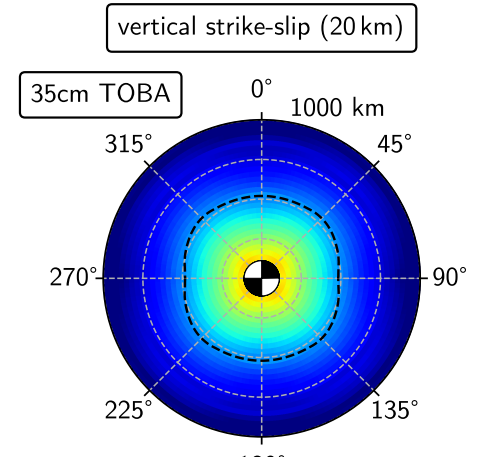

$180^{\circ}$

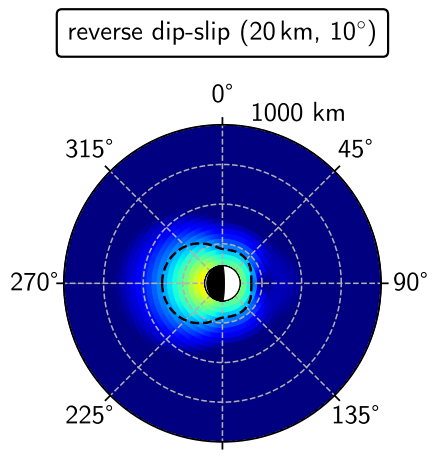

$180^{\circ}$

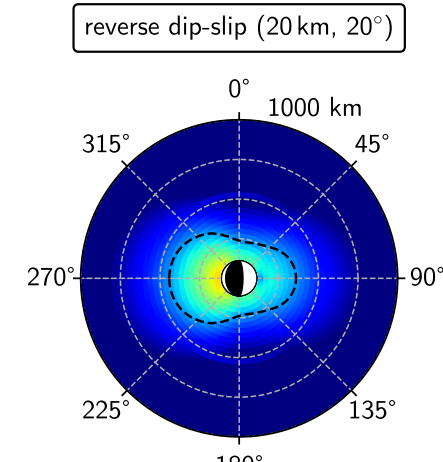

$180^{\circ}$

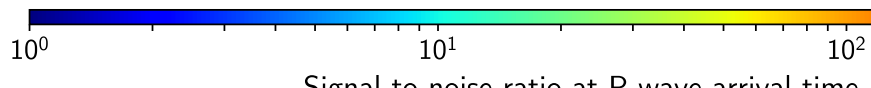

Signal-to-noise ratio at P-wave arrival time

Figure 11. Azimuth-distance distributions of SNR using $35 \mathrm{~cm}$-scale TOBA at the P-wave arrival time of each point. Sum of the SNRs of two horizontal gravity strain components is shown in each color map.

instrumental noise below $0.1 \mathrm{~Hz}$ reduces the SNR at a later time $(t \gtrsim 10 \mathrm{~s})$ at the distant locations.

Thus, the proposed configuration of TOBA can contribute to earthquake detection, though the SNRs are degraded by the lowfrequency noise and noise peaks. The same analysis can be applied to other detectors, to examine the design of the detector sensitivity. The sensitivity of TOBA can be improved by increasing the length of the bars and the laser power of the interferometer (Ando et al., 2010). For example, the fundamental sensitivity limits, such as quantum noise or thermal noise, are ten times lower with a 1 m-scale TOBA.

\section{Appendix B. SNR at 5 seconds after the onset of the fault rupture}

Fig. 12 shows the SNR distribution at 5 seconds after rupture onset. The detectable area is quite limited compared to the detection at 10 $\mathrm{s}$ and to the $\mathrm{P}$ wavefront at $5 \mathrm{~s} \mathrm{(Fig.} \mathrm{3).} \mathrm{This} \mathrm{is} \mathrm{due} \mathrm{to} \mathrm{the} \mathrm{strong} \mathrm{time}$ dependence of the gravity signal amplitude, which is roughly proportional to $t^{6}$ in our model based on a source time function with quadratic onset (Eq. (1)) and a homogeneous half-space (Harms,
2016). Therefore, with the sensor sensitivities considered here, the gravity strain signal cannot be detected much earlier than $10 \mathrm{sec}-$ onds over a sufficiently wide area. 


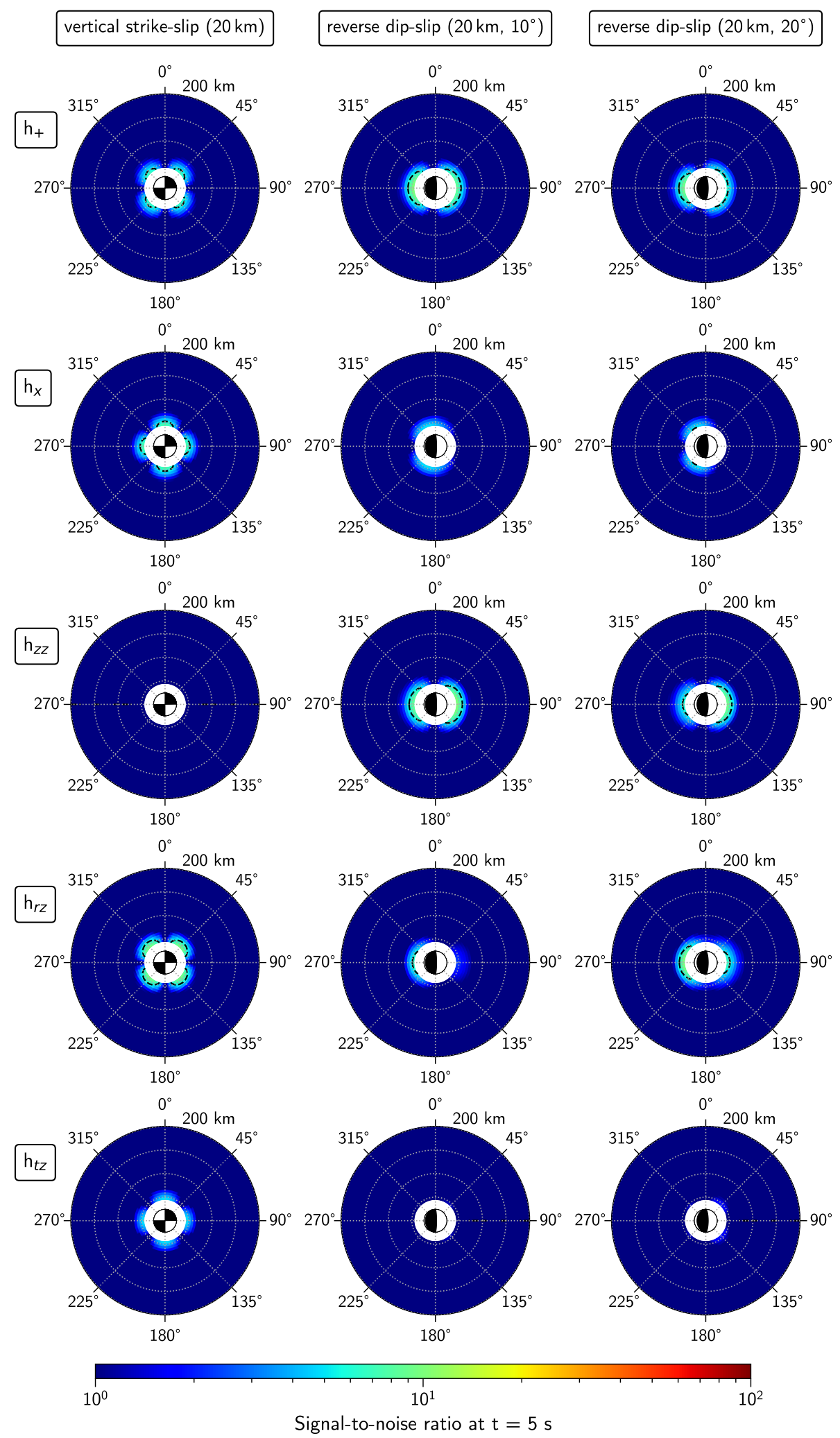

Figure 12. Azimuth-distance distributions of SNR at 5 seconds after rupture onset. Inside the white area $(\lesssim 45 \mathrm{~km})$ seismic P-waves arrive within 5 seconds. The other assumptions are the same as in Fig. 3. 